\title{
Stereotactic Characteristics of the Thalamus Subject to Shape of the Skull: An MRI Study
}

\author{
Maryna Kornieieva ${ }^{1,2 *}$, Iuliia Zhuravlova ${ }^{3}$ and Azmy Hadidy ${ }^{4}$ \\ ${ }^{1}$ Department of Anatomy, Faculty of Human Medicine, Al-Balqa Applied University, Jordan \\ ${ }^{2}$ Department of Anatomy, American University of Caribbean's Medical School, St.Marteen \\ ${ }^{3}$ Department of Anatomy, Trinity School of Medicine, St.Vincent and the Grenadines \\ ${ }^{4}$ Radiology and Nuclear Medicine Department, Jordan University Hospital, Jordan
}

${ }^{*}$ Corresponding author: M Kornieieva, Department of Anatomy, Faculty of Human Medicine, Al-Balqa Applied University, As-Salt 19110, Al-Buhaira Street 31/1, Jordan, Tel: 00962770572363 Email: marykorn15@gmail.com

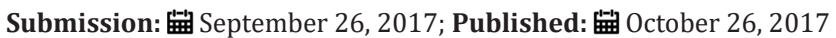

\begin{abstract}
Introduction: The thalamus is a nuclear complex located in the dorsal part of the diencephalon. The wide implementation of deep brain stimulation into minimally invasive neurosurgical practice requires accurate localization of the thalamus. The present study is designed to assess the influence of the individual shape of the skull in relation to the stereotactic characteristics of the thalamus.

Materials and methods: A total of 140 Arab patients underwent routine MRI examination of the head. The perpendicular distances from the outer surface of the skull bones to anterior, lateral and superior borders of the thalamus were analyzed in patients with dolichocephalic, mesocephalic, and brachycephalic shapes of the skull. The maximum-likelihood estimation of the observed variables was generalized to the studied population with the method of robust regression.

Results: The distance from the anterior end of the thalamus to the outer surface of the frontal bone varies from the $81.92 \mathrm{~mm}$ in the group of subjects with the dolichocephalic shape of the skull to $77.92 \mathrm{~mm}$ in the brachiocephalic group, with the middle value obtained in the mesocephalic group. The opposite tendency concerns the distance from the midpoint of the lateral border of the thalamus to the outer surface of the temporal bone.

Conclusion: The distances between the counter sides of the thalamus and the outer surfaces of overlaying bones do correlate with the shape of skull, and may contribute to personalization of preoperative stereotactic analysis and approach.
\end{abstract}

Keywords: Variability; Thalamus; Deep brain stimulation

\section{Introduction}

The thalamus is a paired mass of grey matter situated on both sides of the third ventricle in the dorsal part of the diencephalon that is involved in relaying sensory and motor signals to the relevant cortical areas. The internal medullary lamina divides the nuclei of the thalamus into three anatomical groups: anterior, media land lateral, characterized by specific connections of forming neurons. The anterior and medial thalamic regions are each composed of single nuclei, while the lateral region is subdivided into ventral and dorsal tiers each of which contains a number of sub-nuclei. The prominent caudal part of the thalamus - the pulvinar, overhangs the superior colliculus and brachium and, additionally, is composed of four subgroups of neurons. Having excessive reciprocal connections with both cerebral cortex and midbrain, the neurons of the thalamic nuclei are able to modulate and filter transmitting signals, facilitating functional plasticity of the forebrain [1].

The interest in the thalamus progressively increased through the last decade due to wide implementation of the "deep brain stimulation" methods of minimally invasive neurosurgical practice
[2-5]. Deep brain stimulation is a powerful method capable of modulating a large variety of neurological symptoms for both movement and psychiatric disorders. This procedure is performed "blindly" on the deep structures of the brain of an awake patient, and the success ultimately depends on accuracy of hitting the functional target during surgery. The extended intra operative exploration with the penetration of multiple electrodes is the main source of surgical complications, such as prolongation of surgical time, potential infection, and risk of hemorrhage [6,7].

The accurate stereotactic analysis of the patient's head images and the proper localization of the desired target on the preparatory steps help to reduce the risk factors. The best technique available today for visualization of the deep gray matter brain structures is magnetic resonance imaging (MRI). It allows localization of the thalamus between the body of the fornix, the anterior and posterior cerebral commissures. Then, the stereotactic reference systems are employed to transfer the coordinates of certain targets onto the patient's anatomy [8]. However, the robust stereotactic 
reference systems and modern 3Dneuroanatomical atlases are usually constructed on the basis of a single brain specimen, while localization of the sub-cortical functional targets do vary significantly from patient to patient. Understanding the individual constitution of the head and knowledge of the allowable range of stereo-topographic localization of the thalamus would help related specialists to succeed with the pre-operative stereotactic analysis. The present radiological study is designed to assess the influence of the individual shape of the head on the stereotactic characteristics of the anterior, posterior, lateral and superior boundaries of the thalamus. No previous data related to the topic are found in the literature.

\section{Materials and Methods}

This study was carried out on 140 subjects of Arab descent ( 68 males, 72 female), aged between 26-78 years old, without notable deformations of the skull, neurologic symptoms, or other diseases associated with the central nervous system and head structures, who underwent routine MRI examination of the head under the direct supervision of one of the authors in accordance with the ethical standards. Written informed consents were obtained from all the subjects who agreed to participate in the study. MRI scans were performed in the Radiological Department of the University of Jordan Hospital using a whole-body 3 Tesla MR system (Magnetom Verio; Siemens Medical Solution, Erlangen, Germany).The protocol was identical for all participants and included axial and sagittal T1 and T2-weighted ultra-fast gradient echo sequences (280/2.6 [repetition time ms/echo time ms]; flip angle 15u, 230mm field of view with a 0.10 distant factor).

On sagittal and axial MR images of the head, the longitudinal (LD) and transverse (TD) diameters of the skull were measured from the glabella to the opisthocranion(G-OP) and from the euryon to euryon (EU-EU), respectively, to determine the type of head constitution. The cranial index (CI) was computed in accordance with standard anatomical descriptions as the ratio of TD to LD percentagewise $[9,10]$.

The research group was divided into three subgroups regarding to the value of the $\mathrm{CI}$ :

1) The dolichocephalic group ( $\mathrm{CI}<74.9 \%)$

2) The mesocephalic group (CI in the range 75\%-79.9\%), and

3) The brachycephalic group (CI > 80\%).

On the axial T2 weighted and inversion recovery sequences, the boundaries of the thalamus were distinguished. The anterior border of the thalamus was defined at the posterior point of the inter-ventricular foramen, the lateral border was marked at its contact with the posterior limb of the internal capsule, and the projected backward edge of the pulvinar was considered as the posterior border of the thalamus.

The perpendicular distances from the outer surface of the skull bones to
1) The anterior end of the thalamus

2) The midpoint of the pulvinar and

3) The midpoint of the lateral border of the thalamus was measured using an open source DICOM viewer (Radi Antv.3.4.1).

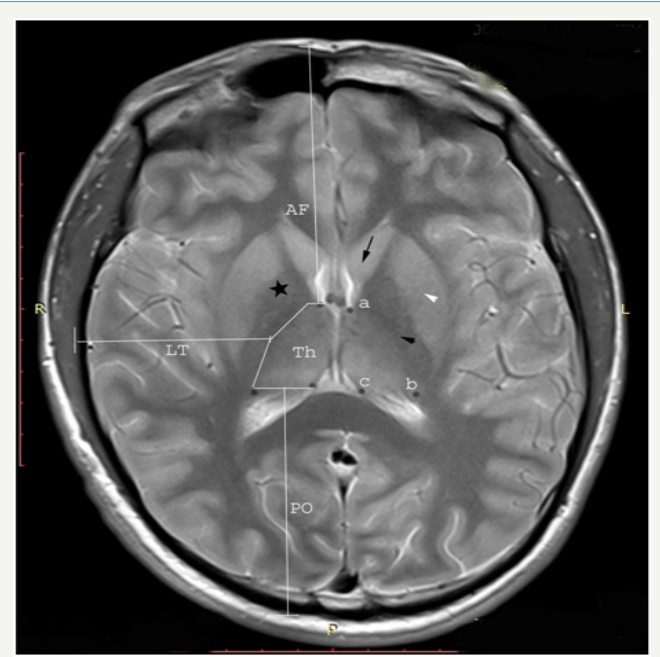

Figure 1: An axial T2-weighted magnetic resonance image demonstrating the anatomical landmarks used to localize the anterior (a) and posterior (b-c) boundaries of the thalamus (Th). The lateral border is distinguished at its conjunction with the posterior limb of the internal capsule (black arrowhead). The measured distances between the anterior end of the thalamus and the outer surface of the frontal bone (AF), the middle of the lateral border and the outer temporal bone (LT), and the midpoint of the posterior border and the outer occipital bone (PO) are marked. The globus pallidus (star), putamen (white arrowhead), and the head of the caudate nucleus (black arrow) are identified.

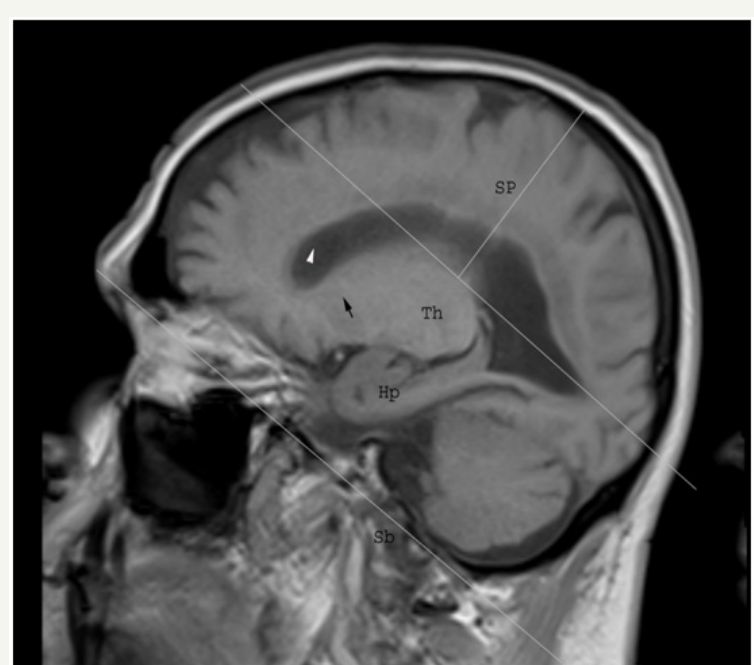

Figure 2: A sagittal T2-weighted magnetic resonance image showing the superior border of the thalamus (Th) along the line reproduced parallel to the skull base plane (Sb), and the distance measured perpendicular to the outer surface of the parietal bone (SP).The head of the caudate nucleus (black arrow), the anterior horn of the lateral ventricle (white arrowhead), and the hippocampus (Hp) are clearly distinguished on the image. 
On the sagittal MRI images, the highest outline of the thalamus was found at the level of the body of the lateral ventricle. The line parallel to the skull base was drawn through the highest point of the superior border of the thalamus, and the perpendicular distance from it to the outer surface of the overlying parietal bone was taken (Figure 1\&2).

The obtained data were computed and analyzed with SPSS for Windows v.21 software. After the calculation of descriptive statistics for males and females in the studied groups, the independent samples t-Test was applied to assess the significance of the group mean differences in the marginal brachiocephalic and dolichocephalic subgroups; the mean value obtained in the mesocephalic group was considered as a test value. Significance of correlation between the measured distances and the CI was analyzed using the Pearson's correlation coefficient; a p value 0.05 and less was considered as statistically significant.

The maximum-likelihood (M) estimation of the observed variables was generalized to the studied population via the method of robust regression. With M-estimation, the initial estimate $b$ was determined by minimizing a particular objective function over all $b$. The Humber's, Tukey's, Hamppel's and Andrews' wave weighting constants $(\mathrm{w})$ were used in computing of the estimation equation:

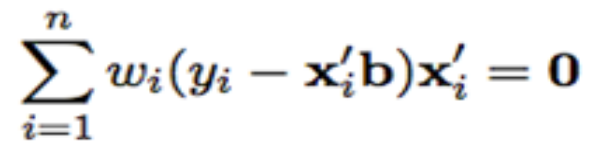

Where $\mathrm{x}$ is a model matrix, with $\mathrm{x}_{\mathrm{i}}$ as its $\mathrm{i}^{\text {th }}$ row, $\mathrm{n}$-number of observations, and $\mathrm{w}_{\mathrm{i}}$ is the current weight constant [11].

\section{Results}

The statistic analysis of the obtained data shows the significant difference of the variables in the studies of cranial subgroups (Table 1). Thus, the distance from the anterior end of the thalamus to the outer surface of the frontal bone (AF) varies from the $81.92 \mathrm{~mm}$ in the group of subjects with the dolichocephalic shape of the skull (DC) to $77.92 \mathrm{~mm}$ in the brachycephalic group (BC), with the middle value obtained in the mesocephalic group (MC). The distance from the posterior border of the pulvinar to the outer surface of the occipital bone (PO) is also maximal in the DC group and is equal to $73.75 \mathrm{~mm}$, while the minimal value is possessed by the BC subjects $(63.75 \mathrm{~mm})$. The opposite tendency concerns the distance from the midpoint of the lateral border of the thalamus to the outer surface of the temporal bone (MT). It is larger in the BC group (55.05mm), than in the MC $(50.09 \mathrm{~mm})$ and the $\mathrm{DC}(46.77 \mathrm{~mm})$ ones. The considered distances slightly dominated in males in comparison with females.

Table 1: General and sex-related descriptive statistics among the subjects with the mesocephalic (MC), brachycephalic (BC) and dolichocephalic (DC) shape of the skull with independent samples t-test for equality of means and Pearson correlation.

\begin{tabular}{|c|c|c|c|c|c|c|c|c|}
\hline Variables & $\begin{array}{c}\text { Studied } \\
\text { Subgroups }\end{array}$ & $\begin{array}{c}\text { Mean, mm } \\
\quad( \pm \text { SD })\end{array}$ & $\begin{array}{l}\text { Mean, mm } \\
\text { males }( \pm \\
\text { SD) }\end{array}$ & $\begin{array}{l}\text { Mean, mm } \\
\text { females ( } \pm \\
\text { SD) }\end{array}$ & $\begin{array}{l}\text { Pearson } \\
\text { correlation } \\
\text { with CI }\end{array}$ & $\begin{array}{c}\text { Significance of } \\
\text { the Correlation } \\
\text { (2-tailed) }\end{array}$ & $\begin{array}{l}t \text {-Test for } \\
\text { Equality of } \\
\text { Means1 }\end{array}$ & $\begin{array}{c}\text { Significance } \\
\text { of the } t \text {-test } \\
\text { (2-tailed) }\end{array}$ \\
\hline \multirow{6}{*}{$\mathrm{AF}$} & \multirow{2}{*}{$\mathrm{MC}$} & 79.2038 & \multirow{2}{*}{$\begin{array}{c}80.2682 \\
(3.38925)\end{array}$} & \multirow{2}{*}{$\begin{array}{c}78.4233 \\
(4.50752)\end{array}$} & \multirow{2}{*}{$-0.470^{* *}$} & \multirow{2}{*}{0} & \multirow{2}{*}{0.007} & \multirow{2}{*}{0.995} \\
\hline & & -4.13886 & & & & & & \\
\hline & \multirow{2}{*}{$\mathrm{BC}$} & 77.925 & 79.0846 & 76.1842 & \multirow{2}{*}{$0.371^{*}$} & \multirow{2}{*}{0.037} & \multirow{2}{*}{$-3.444^{* *}$} & \multirow{2}{*}{0.002} \\
\hline & & -4.99477 & -5.73786 & -4.11059 & & & & \\
\hline & \multirow{2}{*}{ DC } & 81.925 & 83.5 & 80.7526 & \multirow{2}{*}{$-0.674^{*}$} & \multirow{2}{*}{0.014} & \multirow{2}{*}{$3.320^{* *}$} & \multirow{2}{*}{0} \\
\hline & & -4.99477 & -3.74833 & -3.68757 & & & & \\
\hline \multirow{6}{*}{ PO } & \multirow{2}{*}{ MC } & 67.0827 & 68.8864 & 65.76 & \multirow{2}{*}{$-0.325^{* *}$} & \multirow{2}{*}{0.001} & \multirow{2}{*}{-0.28} & \multirow{2}{*}{0.781} \\
\hline & & -5.60206 & -6.18464 & -4.81818 & & & & \\
\hline & \multirow{2}{*}{$\mathrm{BC}$} & 63.7594 & 63.5538 & 62.9 & \multirow{2}{*}{$-0.531^{*}$} & \multirow{2}{*}{0.04} & \multirow{2}{*}{$-6.246^{* *}$} & \multirow{2}{*}{0} \\
\hline & & -3.20643 & -3.80955 & -2.82489 & & & & \\
\hline & \multirow{2}{*}{ DC } & 73.7594 & 74.1 & 72.0211 & \multirow{2}{*}{$0.401^{*}$} & \multirow{2}{*}{0.023} & \multirow{2}{*}{$6.324^{* *}$} & 0 \\
\hline & & -3.20643 & -2.97686 & -3.02702 & & & & 0 \\
\hline & $M C$ & 50.0925 & 50.9045 & 49.2303 & ( $754 * *$ & & 1517 & 0835 \\
\hline & WIC & -2.34146 & -2.60521 & -2.16338 & 0.154 & 0.002 & $1.51 /$ & 0.035 \\
\hline MT & $B C$ & 55.0567 & 58.4818 & 52.9529 & $0528 * *$ & 0004 & $2304 *$ & \\
\hline M11 & $\mathrm{BC}$ & -4.36851 & -2.99327 & -2.97513 & $0.528^{\pi x}$ & 0.004 & $2.304^{\star}$ & 0.029 \\
\hline & $\mathrm{DC}$ & 46.7761 & 47.1231 & 46.1111 & $0423 *$ & 0 & 4 & 0 \\
\hline & $\mathrm{DC}$ & -4.36851 & -2.51202 & -2.13572 & $0.423^{*}$ & 0.009 & $-4.298^{* a}$ & 0 \\
\hline & $M C$ & 62.9077 & 64.3545 & 61.8467 & $-276^{* * *}$ & 0005 & - 139 & 089 \\
\hline SP & WV & -4.78473 & -5.92844 & -3.47243 & $-.2 / 6$ & 0.005 & -0.139 & 0.89 \\
\hline 01 & $B C$ & 62.9821 & 62.1154 & 62.7842 & 0193 & 0291 & - 741 & 0864 \\
\hline & BC & -3.17397 & -3.37709 & -4.00365 & 0.193 & 0.291 & $-0 . / 41$ & 0.864 \\
\hline
\end{tabular}




\begin{tabular}{|l|l|l|l|l|l|l|l|l|}
\hline & \multirow{2}{*}{ DC } & 62.9821 & 63.0885 & 62.7737 & \multirow{2}{*}{-0.189} & 0.299 & 0.969 \\
\cline { 3 - 5 } & & -3.17397 & -4.0883 & -3.71077 & & 0.734 \\
\hline
\end{tabular}

1The mean values of a variable in $\mathrm{BC}$ and $\mathrm{DC}$ subgroups were considered in the t-test, while the respective value in MC group was taken as a test-value.

Significance levels: ${ }^{* *} \mathrm{p}<0.01 ;{ }^{*} \mathrm{p}<0.5$

Table 2: Robust regression for estimation of the maximum-likelihood topography of the thalamus subject to shape of the skull

\begin{tabular}{|c|c|c|c|c|c|c|c|c|}
\hline \multirow{2}{*}{ Variables } & \multirow{2}{*}{$\begin{array}{l}\text { Studied } \\
\text { Subgroups }\end{array}$} & \multicolumn{2}{|c|}{$\begin{array}{l}\text { 95\% Confidence Interval for } \\
\text { Mean }\end{array}$} & \multirow{2}{*}{ Std. Error } & \multirow{2}{*}{$\begin{array}{c}\text { Huber's } \\
\text { M-Estimatora }\end{array}$} & \multirow{2}{*}{$\begin{array}{c}\text { Tukey's } \\
\text { Biweightb }\end{array}$} & \multirow{2}{*}{$\begin{array}{c}\text { Hampel's } \\
\text { M-Estimatorc }\end{array}$} & \multirow{2}{*}{$\begin{array}{c}\text { Andrews' } \\
\text { Waved }\end{array}$} \\
\hline & & Lower Bound & Upper Bound & & & & & \\
\hline \multirow{3}{*}{$\mathrm{AF}$} & MC & 77.7078 & 81.8672 & 0.177454 & 78.9563 & 78.8979 & 79.0721 & 78.8989 \\
\hline & $\mathrm{BC}$ & 76.1242 & 78.7258 & 0.088296 & 78.3561 & 78.3412 & 78.2679 & 78.3381 \\
\hline & DC & 79.7328 & 83.3797 & 0.064888 & 80.2892 & 80.2929 & 80.2572 & 80.2909 \\
\hline \multirow{3}{*}{ PO } & MC & 64.9145 & 69.4542 & 0.11294 & 66.6523 & 66.4335 & 66.6659 & 66.4313 \\
\hline & $\mathrm{BC}$ & 61.6033 & 63.9154 & 0.056682 & 64.2239 & 64.3456 & 64.1289 & 64.354 \\
\hline & DC & 69.5675 & 75.7262 & 0.052921 & 70.6092 & 70.5932 & 70.6567 & 70.5928 \\
\hline \multirow{3}{*}{ MT } & MC & 48.2899 & 50.9744 & 0.041048 & 50.0282 & 49.9623 & 49.9748 & 49.9623 \\
\hline & $\mathrm{BC}$ & 51.7514 & 59.2129 & 0.059982 & 50.5767 & 50.5154 & 50.678 & 50.5068 \\
\hline & DC & 45.8146 & 48.5647 & 0.142646 & 47.9236 & 47.9516 & 47.8616 & 47.9504 \\
\hline \multirow{3}{*}{ SP } & MC & 60.431 & 65.0378 & 0.088423 & 62.3272 & 61.8229 & 62.0221 & 61.824 \\
\hline & $\mathrm{BC}$ & 62.5125 & 63.8535 & 0.265749 & 62.6059 & 62.5827 & 62.5632 & 62.5826 \\
\hline & DC & 62.1185 & 64.8721 & 0.267505 & 63.8104 & 63.8069 & 63.6484 & 63.806 \\
\hline
\end{tabular}

a- weighting constant is 1.339 ; b- weighting constant is 4.685 ; c- weighting constants are $1.700,3.400$, and 8.500 ; d- weighting constant is $1.340^{*} \mathrm{pi}$

The Pearson correlation analysis reveals statistically significant correlation of the stereotactic characteristics of the thalamus with the individual shape of skull (Table 1). Especially strong correlation of the thalamic topography with the $\mathrm{CI}$ is disclosed in the MC group. On the other hand, the SP interval shows no associations with the shape of skull. The obtained results of the paired t-test confirm the significance of mean differences in the marginal cranial groups (DC and $\mathrm{BC}$ ), than the respective mean of the MC group taken for the test-value. However, the interval from the superior border of the thalamus to the outer surface of the parietal bone is approximately equal in all studied groups (Table 1). The maximum-likelihood estimation of the observed variables was generalized to the studied population by the M-estimation method of robust regression. The expected parameters calculated with the Humber's, Tukey's biweight, Hampel's and Andrews' wave estimators are presented in Table 2.

\section{Discussion}

The first exploring electrode was successfully centered in the thalamus of a patient with Parkinson's tremor in 1997 [2]. Although the target for non-invasive treatment of such patients was recently shifted to the subthalamic nucleus and the internal globus pallidus $[3,4]$, the wide connections and functional variability of the thalamic nuclei are still under rapt attention of specialists. Thus, the ventro intermediate thalamic nucleus is an approved point for stimulation in case of non-Parkinsonian essential tremor, while the anterior, medial and posterior thalamic regions undergo experimental study now as the functional targets for the relief of the symptoms of epilepsy, schizophrenia, and incessant pain, respectively $[5,12]$.

One of the main obstacles of this perspective minimally invasive neurosurgical technique is the anatomical variability of the thalamus and its nuclei. Probabilistic diffusion tractography of cortico-thalamic connections carried out by Johansen-Berg et al. [13] have proved significant inter-individual variability of thalamic anatomy appeared as "fuzziness" of borders of functional areas of the thalamus obtained during the group-mapping, which involved not only the internal segmentation of the thalamus into groups and subgroups, but also the connected cortical areas. In their research, Anderson \& Mullins [14] have linked a wide range of behavioral symptoms following the deep brain stimulation such as depression, mania, and hallucinations with hyper stimulation of these overlapped cortical areas. Although the surgical complications are less common, the study of Fenoy \& Simpson [15] shows that longterm prognosis of the intra-ventricular hemorrhage (3.4\%), wound infection $(1.7 \%)$, or malposition of hardware $(1.7 \%)$ may be highly unfavorable for patients.

Applied in this study 3Tesla MRI technique is now considered the best visualizing modality allowing clear identification of the boundaries of the sub-cortical grey matter [5]. Urgent interest to subdivisions of the thalamus inspired Bender et al. [16] to optimize and modernize protocols trying to enable direct visualization of the main nuclear groups of the thalamus and to improve targeting with coordinates from classic anatomical atlases. However, 
the subdivisions of thalamic gray matter are still unclear and interpretation of images may be biased. Thorough understanding of general principles of thalamic topography and effective estimation of individual variability of the thalamus on the basis of head constitution could help to individualize depiction of thalamic substructures and eliminate a number of misinterpretations.

The published results of systematic studies on the anatomical variability of the thalamus are quite controversial. On the basis of micro- and macroscopic study of 120 brains in different age groups of subjects, Maslovskii [17] concluded that the anterior, medial and lateral nuclei of the thalamus are individually variable and do not underlay any of regularities, having no correlation with sex, age, or size of the brain. On the other hand, the investigation of Sen et al. [18] showed that transverse and vertical diameters of the thalamus slightly dominate in females, while antero-posterior length is larger in males with no correlation with age. Specific signs of atrophy of the thalamic mass, and histological changes in the dorsal nuclei and pulvinar were noticed in patients with Huntington's disease and schizophrenia, respectively $[12,19,20]$. Although the direct measurements of the thalamic dimensions were not conducted in our study, the obtained results show that the distances between the counters of the thalamus and the outer surfaces of overlaying bones do correlate with the shape of skull, and may contribute to personalization of preoperative stereotactic analysis of the thalamus, mapping of its nuclei, and the planning of surgical approach. The sex difference in the thickness of the skull bones leads to slight dominance of the considered parameters in males and must be counted as well.

Utilization of the individual parameters of the patient's skull by regression analysis facilitates the individual approach to stereotactic analysis of the thalamus. Due to geographic dependency of the craniological characteristics of the skull revealed by Boas [21], the estimates obtained from the specific robust regression model should be applied only in populations where the measurements were taken.

\section{References}

1. Snell R (2010) Clinical Neuroanatomy. $7^{\text {th }}$ Ed Lippincott Williams \& Wilkins Philadelphia, USA.

2. Benabid A (2003) Deep brain stimulation for Parkinson's disease. Curr Opin Neurobiol 13: 696-706.

3. Fukuda M, Barnes A, Simon E, Holmes A, Dhawan V, et al. (2004) Thalamic stimulation for parkinsonian tremor: correlation between regional cerebral blood flow and physiological tremor characteristics. Neuro Image 21: 608-615.

4. Riley J, Boulis N (2011) Emerging Targets for Stimulation-Refractory Movement Disorders. The Open Neurosurgery Journal 4(S1-M5): 53-61.
5. Jiltsova E, Moettoenen T, Fahlstroem M, Haapasalo J, Taehtinen T, et al. (2016) Imaging of Anterior Nucleus of Thalamus Using 1.5T MRI for Deep Brain Stimulation Targeting in Refractory Epilepsy. Neuromodulation 19(8): 812-817.

6. Hariz M (2002) Complications of Deep Brain Stimulation Surgery. Mov Disord 17(S3): 162-166.

7. Voges J, Waerzeggers $Y$, Maarouf M, Lehrke R, Koulousakis A, et al. (2006) Deep-brain stimulation: long-term analysis of complications cause by hardware and surgery-experiences from a single centre. J Neurol Neurosurg Psychiatry 77(7): 868-872.

8. Ooteman W, Cretsinger K. (2002) Thalamus Tracing Guidelines.

9. Bannister M (1995) Skeletal System. In: Williams P, Dyson M, Dussak JE, Bannister LH, Berry MM (Eds.), Gray's Anatomy. (38 ${ }^{\text {th }}$ edn), Elsevier Churchill Livingston, London, UK.

10. Kolar J, Salter E (1997) Craniofacial Anthropometry, Practical measurement of the head and face for clinical, surgical and research use. Charles C Thomas Springfield, Illinois, USA.

11. Fox J (1997) Applied Regression Analysis, Linear Models and Related Methods. ( $3^{\text {rd }}$ edn), Sage Publication, USA.

12. Mavridis I (2014) Human mediodorsal thalamic nucleus as a potential target for deep brain stimulation: review of the literature and anatomical considerations. OA Anatomy 2(1): 1.

13. Johansen-Berg H, Behrens T, Sillery E, Ciccarelli O, Thompson A, et al. (2005) Functional anatomical validation and individual variation of diffusion tractography-based segmentation of the human thalamus. Cereb Cortex 15(1): 31-39.

14. Anderson K, Mullins J (2003) Behavioral changes associated with deep brain stimulation surgery for Parkinson's disease. Curr Neurol Neurosci Rep 3(4): 306-313.

15. Fenoy A, Simpson R (2014) Risks of common complications in deep brain stimulation surgery: management and avoidance. Journal of Neurosurgery Jan 120(1): 132-139.

16. Bender B, Manz C, Korn A, Nagele T, Klose U (2011) Optimized 3D Magnetization-Prepared Rapid Acquisition of Gradient Echo: Identification of Thalamus Substructures at 3T. AJNR Am J Neuroradiol 32(11): 2110-2115.

17. Maslovskii S (1984) Individual anatomic variability of particular thalamic nuclei in children and adolescents with respect to stereotaxic coordinates. Arkh Anat Gistol Embriol 87(7): 18-26.

18. Sen F, Ulubay H, Ozeksi P, Sargon M, Tascioglu B (2005) Morphometric measurements of the thalamus and interthalamic adhesion by MRI imaging. Neuroanatomy 4: 10-12.

19. Kassubek J, Juengling F, Daniel Ecker D, Bernhard L (2005) Thalamic Atrophy in Huntington's Disease Co-varies with Cognitive Performance: A Morphometric MRI Analysis. Cereb Cortex 15(6): 846-853.

20. Csernansky J, Mathew K, Schindler N, Lei-Wang R, Mohktar G, et al. (2004) Abnormalities of Thalamic Volume and Shape in Schizophrenia. Am J Psychiatry 161(5): 896-902.

21. Boas F (1899) The cephalic index. American Anthropologist 1(3): 448461. 